\title{
Experimental Quenching of Harmonic Stimuli: Universality of Linear Response Theory
}

\author{
Paolo Allegrini, ${ }^{1}$ Mauro Bologna, ${ }^{2,3}$ Leone Fronzoni, ${ }^{1}$ Paolo Grigolini, ${ }^{1,2,4}$ and Ludovico Silvestri ${ }^{1,5}$ \\ ${ }^{1}$ Dipartimento di Fisica “E. Fermi,” Università di Pisa and INFM CRS-SOFT, Largo Pontecorvo 3, 56127 Pisa, Italy \\ ${ }^{2}$ Center for Nonlinear Science, University of North Texas, P.O. Box 311427, Denton, Texas 76203, USA \\ ${ }^{3}$ Instituto de Alta Investigación, Universidad de Tarapacá-Casilla 6-D Arica, Chile \\ ${ }^{4}$ Istituto dei Processi Chimico Fisici del CNR Area della Ricerca di Pisa, Via G. Moruzzi 1, 56124 Pisa, Italy \\ ${ }^{5}$ L.E.N.S., University of Florence, via Nello Carrara 1, 50019 Sesto Fiorentino (FI), Italy \\ (Received 26 May 2009; revised manuscript received 16 June 2009; published 15 July 2009)
}

\begin{abstract}
We show that liquid crystals in the weak turbulence electroconvective regime respond to harmonic perturbations with oscillations whose intensity decay with an inverse power law of time. We use the results of this experiment to prove that this effect is the manifestation of a form of linear response theory (LRT) valid in the out-of-equilibrium case, as well as at thermodynamic equilibrium where it reduces to the ordinary LRT. We argue that this theory is a universal property, which is not confined to physical processes such as turbulent or excitable media, and that it holds true in all possible conditions, and for all possible systems, including complex networks, thereby establishing a bridge between statistical physics and all the fields of research in complexity.
\end{abstract}

DOI: 10.1103/PhysRevLett.103.030602

The linear response theory (LRT) [1] is a theoretical tool of general interest in physics. In spite of some criticism [2], the experimental work done over a time span of about 52 years has not revealed any breakdown of the theory. There is general agreement that the LRT is one of the fundamental accomplishments of statistical physics. In addition to affording an invaluable guideline for experimental investigation in condensed matter, the LRT led Lee [3] (see also [4]) to the definition of an inner operator product and hence to the foundation of an operator basis set that made it possible for him to design a rigorous, efficient, and versatile approach to the relaxation of Hamiltonian systems [5]. Unfortunately, no general theory exists yet to extend in the same elegant manner the LRT from equilibrium to nonequilibrium conditions [6].

The conventional LRT rests on two basic assumptions: (i) The time evolution of the system variable $\xi_{S}$ is driven by Hamiltonian operators (Liouville equation); (ii) The external perturbation $\xi_{P}$ has the effect of making the system depart from canonical equilibrium so weakly as to render the linear response function $\chi\left(t, t^{\prime}\right)$ identical to the derivative of the correlation function $C\left(t, t^{\prime}\right)$ [7],

$$
\chi\left(t, t^{\prime}\right)=\frac{d}{d t^{\prime}} C\left(t, t^{\prime}\right),
$$

where $C\left(t, t^{\prime}\right)=C\left(t-t^{\prime}\right)$ is stationary. The response is

$$
\left\langle\xi_{S}(t)\right\rangle=\epsilon \int_{0}^{t} d t^{\prime} \chi\left(t, t^{\prime}\right) \xi_{P}\left(t^{\prime}\right),
$$

with $\epsilon<1$ being the interaction strength.

The recent literature on non-Poisson renewal processes is raising increasing interest on the action of nonergodic renewal events [8-10], with a wide set of applications, ranging from quantum mechanics [11] to the brain dynam-
PACS numbers: 05.60.Cd, 02.50.Ey, 47.52.+j, 61.30.-v

ics [12], thereby casting deep doubt on the possibility of using the conventional LRT to study the effects of perturbation in these cases. This is so for two main reasons: (i) It is very difficult, if not impossible [13], to describe the time evolution of the event-driven systems by means of Hamiltonian operators (classical or quantum Liouville equation); (ii) It is not yet well understood how to use the linear response structure of Eq. (2) when a stationary correlation function is not available, in spite of the fact that some prescriptions already exist $[14,15]$. These are probably the reasons why Sokolov and Klafter [16,17] coined the term "death of linear response" to denote the fading away response of a complex system to a harmonic stimulus. This interesting phenomenon raised the interest of other researchers and also a debate on the best way to generate it with surrogate sequences [18-20].

The purpose of this Letter is twofold: we give the first experimental evidence of this interesting effect, task No. 1 , but we argue that surprisingly the LRT is in action also in this case, task No. 2. We use real experiments on liquid crystals, not only to support our theoretical arguments, but, more importantly, to make our conclusions accessible to an audience of readers as wide as possible. Our arguments do not require a Hamiltonian formalism: this is not a limitation, but rather a significant extension of the LRT that is expected to apply not only to physical systems but to neurophysiological and sociological processes as well [21], thereby involving an interdisciplinary audience.

The guidelines for this wide audience of readers to understand the new LRT are indicated by the following three steps: preparation, perturbation, and experiment.

Preparation.-There are systems, and liquid-crystals electroconvective belongs to this group, whose nonequilibrium nature is determined by a cascade of renewal 
events with a rate $R(t)$ decreasing in time as

$$
R(t) \propto \frac{1}{t^{2-\mu}},
$$

after a proper experimental preparation. Equation (3) is a well-known prediction of renewal theory [22] that associates the relaxation $\Psi(t) \propto 1 / t^{\mu-1}$, with $1<\mu<2$, to the production of events with a rate decreasing in time. In the experiment studied in this Letter, $R(t)$ is generated by the dynamics of interacting defects that are prepared at a time preceding the application of a weak perturbation to the system $S$. In the absence of perturbation, $\left\langle\xi_{S}\right\rangle$ is time independent, in spite of the perennial out-of-equilibrium condition represented by the ever drifting quantity $R(t)$ ensuing preparation.

Perturbation.-The external stimulus $\xi_{P}(t)$, even though not coupled to $\xi_{S}$ in a Hamiltonian way, nevertheless affects the event dynamics according to the value of the variable $\xi_{S}(t)$, thereby generating an indirect effect on $\xi_{S}$, and consequently a time-varying bias $\left\langle\xi_{S}(t)\right\rangle$.

Experiment.-The experiment on liquid crystals of this Letter supports the following theoretical prescription

$$
\left\langle\xi_{S}(t)\right\rangle=C R(t) \cos (\omega t+\phi),
$$

with $C$ and $\phi$ as fitting parameters, and $\mu$ of $R(t)$ determined, as we shall see hereby, by the experiment itself. Equation (4) admits a simple and intuitive explanation, as it resembles the response to perturbation of Poisson processes (stochastic resonance [21,23]) with the provision of taking into account the fact that the cascade of events generated by preparation fades away with time.

We study the response to harmonic perturbations of a sample of a nematic liquid crystal (NLC) in a weakly turbulent regime. The experimental setup we used is a very simple and standard one [24]: The nematic (methoxybenzylidene butylaniline, or MBBA) is sandwiched between two glass plates coated with indium oxide to allow electrical conductivity, and an AC potential of frequency $40 \mathrm{~Hz}$ is applied throughout the sample. The glass cell containing NLC has a square base of $1.00 \pm 0.01 \mathrm{~cm}$ side, and a thickness of $25 \pm 1 \mu \mathrm{m}$, leading to an aspect ratio of $400 \pm 17$, and the inner walls are rubbed in order to force planar alignment [25]. This cell is observed through a polarizer microscope: In this configuration, the transmitted light intensity is locally dependent on the orientation field of molecules because of the birefringent nature of NLC. In all the experiments described here, the sample was kept at a constant temperature of $21.000 \pm 0.004^{\circ} \mathrm{C}$, and the illumination lamp was stabilized by a photodiode-driven feedback circuit. When the applied voltage $V$ reaches a critical threshold $V_{C}=19.7 \pm 0.1 \mathrm{~V}$, an electro-hydrodynamic instability sets up, and the molecules self-organize into convective rolls known as Williams domains [26]. Increasing the electric field, several defects appear in this pattern with complicated dynamics of defect births and deaths [27]. For further higher voltages, other turbulent regimes are observed, giving rise to the complex scenarios reviewed in [28]. We focus our attention on electroconvective slightly above $V_{C}$, in the so-called defect-mediated turbulence. As already pointed out in [29], the dynamics of the defects in this regime are regulated by their survival probability, whose power-law behavior in turn affects the slope of the observed $1 / f^{\eta}$ spectrum of fluctuations in the transmitted light intensity, with $\eta=3-\mu$. The system variable $\xi_{S}(t)$ is therefore identified with this intensity, proportional to the number of defects at time $t$ [29], and is measured by focusing the light coming from the microscope to a photodiode, connected to a PC via a data acquisition device.

The experimental observation of Eq. (4) is realized in two steps. In the former phase, corresponding to $V=$ $24.0 \pm 0.1 \mathrm{~V}$ and $\mu>3$, we bring the system to a fully developed turbulent regime so as to destroy all the structures. After $20 \mathrm{~s}$, we move to the latter phase, of defectmediated turbulence, by setting $V_{1}=20.4 \pm 0.1 \mathrm{~V}$, yielding, as we shall see hereby, $\mu \approx 1.5$. We realize the external perturbation in the latter phase by modulating $V$. The two phases generate different equilibrium values for transmittivity. Therefore, in addition to (4), we must take into account also the free regression to equilibrium

$$
\left\langle\xi_{S}(t)\right\rangle=\langle\xi(0)\rangle \Psi(t)+C R(t) \cos (\omega t+\phi) .
$$

To derive the form of Eq. (4), we perturb the system with $\cos (\omega t)$ and $-\cos (\omega t)$, and we evaluate

$$
\Lambda^{ \pm}(t)=\frac{\left\langle\xi_{S}(t)\right\rangle_{+} \pm\left\langle\xi_{S}(t)\right\rangle_{-}}{2}
$$

where $\left\langle\xi_{S}(t)\right\rangle_{+}$and $\left\langle\xi_{S}(t)\right\rangle_{-}$are the responses to $\cos (\omega t)$ and $-\cos (\omega t)$, respectively. Note that $C$ is proportional to $\epsilon \equiv \Delta V /\left(V_{1}-V_{C}\right)=0.025, \Delta V$ being the amplitude in volts of the harmonic perturbation. To obtain ensemble averages \langle\rangle , we repeat the preparation and perturbation procedures about 300 times for each measurement, and

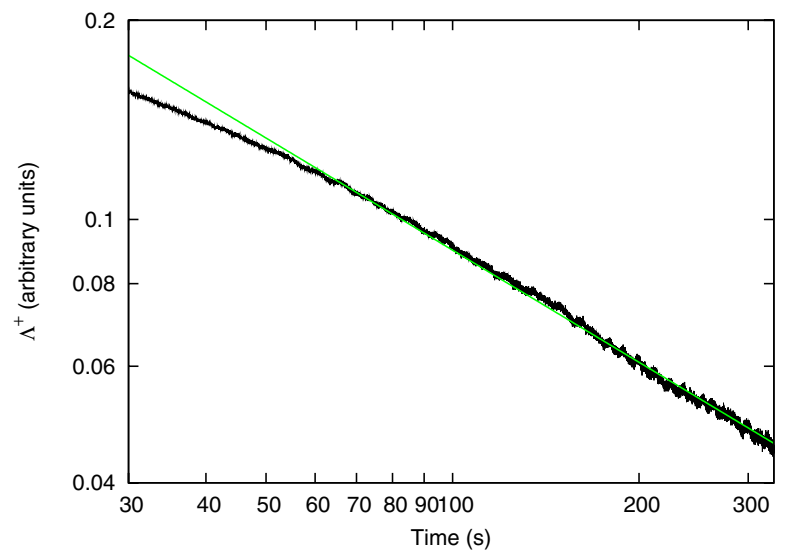

FIG. 1 (color online). $\Lambda^{+}(t)$; green (continuous) line is $t^{1-\mu}$ with $\mu=1.56$. 




FIG. 2 (color online). $\quad \Lambda^{-}(t)$ as a function of time. The almost indistinguishable green (continuous) line is Eq. (5), with frequency $\omega / 2 \pi=0.05 \mathrm{~Hz}$ and fitting parameters $C=0.41, \phi=$ -0.20 .

then we average. $\Lambda^{+}$and $\Lambda^{-}$are shown in Figs. 1 and 2, respectively. The result of Fig. 1 allows us to determine the power index $\mu(=1.56 \pm 0.03$ in this case) [29]. Thus, the very good agreement between the fitting formula of Eq. (4) and the experimental results of Fig. 2 requires only two fitting parameters, $\phi$ and $C$.

After the accomplishment of task No. 1, let us now address task No. 2: How to derive Eq. (4) from a LRT theory? We make the natural conjecture that the new LRT is realized by one of the two choices

$$
\begin{gathered}
\chi\left(t, t^{\prime}\right)=\frac{d}{d t^{\prime}} C\left(t, t^{\prime}\right) ; \\
\chi\left(t, t^{\prime}\right)=-\frac{d}{d t} C\left(t, t^{\prime}\right) .
\end{gathered}
$$

The discussion of which between the two is the most convenient choice was made in Refs. $[14,15])$, and in the earlier work of [30] as well. Here, we show how to relate these choices to $R(t)$ of Eq. (4): An essential step of our experimental-based assessment of the non-stationary LRT. The LRT of a process whose dynamics are dominated by events rather than being driven by the Hamiltonian of the system $S$, which is unknown to us, must be based on the information on the events produced by the system. The central prescription is given by the age-specific rate of event production [31]

$$
g(t)=\frac{\psi(t)}{\Psi(t)}
$$

where $\psi(t)$ is the waiting-time distribution density, related to $\Psi(t)$ by $\Psi(t) \equiv \int_{t}^{\infty} \psi\left(t^{\prime}\right) d t^{\prime}$, yielding, under the normalization condition $\Psi(0)=1$,

$$
\Psi(t)=\exp \left(-\int_{0}^{t} g\left(t^{\prime}\right) d t^{\prime}\right)
$$

In Poisson processes, $g(t)=g$ is time independent so that $\Psi(t)=\exp (-g t)$. The fully turbulent regime produces a high rate of events of this latter kind, but the transition to the defect-mediated turbulent regime makes this high $g$ decrease in time.

The simplest model for inverse-power-law $\Psi(t)$ is

$$
g(t)=\frac{r_{0}}{1+r_{1} t},
$$

which, using Eq. (10), yields

$$
\Psi(t)=\left(\frac{T}{t+T}\right)^{\mu-1},
$$

with $T \equiv 1 / r_{1}$ and $\mu=1+r_{0} / r_{1}$.

It is important to notice that the renewal nature of the process leads us to replace the deterministic function $g(t)$ with the stochastic rate $r(t)$ defined by

$$
r(t)=g\left(t-t_{i}\right)
$$

where $t_{i}$ is the random time of occurrence of the last event prior to time $t$. Thus, the survival probability $\Psi$ depends on the time $t^{\prime}$ at which observation begins

$$
\begin{aligned}
\Psi\left(t, t^{\prime}\right) & =\left\langle\int_{0}^{t^{\prime}} d t^{\prime \prime} R\left(t^{\prime \prime}\right) e^{-\int_{t^{\prime \prime}}^{t} r(\tau) d \tau}\right\rangle \\
& =\int_{0}^{t^{\prime}} d t^{\prime \prime} R\left(t^{\prime \prime}\right) e^{-\int_{t^{\prime \prime}}^{t} g(\tau) d \tau} \\
& =\int_{0}^{t^{\prime}} d t^{\prime \prime} R\left(t^{\prime \prime}\right) \Psi\left(t-t^{\prime \prime}\right) .
\end{aligned}
$$

In the case where the laminar region between two consecutive events, occurring at times $t_{i}$ and $t_{i+1}$, is filled with values $\xi_{S}$ drawn from a distribution with finite width, it is shown [32] that $C\left(t, t^{\prime}\right)$ reduces to $\Psi\left(t, t^{\prime}\right)$, hence

$$
\chi\left(t, t^{\prime}\right)=R\left(t^{\prime}\right) \Psi\left(t-t^{\prime}\right)
$$

$$
\chi\left(t, t^{\prime}\right)=\psi\left(t, t^{\prime}\right)=\int_{0}^{t^{\prime}} d t^{\prime \prime} R\left(t^{\prime \prime}\right) \psi\left(t-t^{\prime \prime}\right)+\psi(t),
$$

which show explicitly as the choices of Eqs. (7) and (8) depend on $R(t)$. The authors of Refs. [7,16,17,19] followed Sokolov [33], whose theory was proved $[14,15]$ to yield the choice of Eq. (15). The rationale for this choice is that the perturbation does not influence the event occurrence time, but only the drawing of the variable $\xi_{S}$ to fill the time intervals between two consecutive events. We refer to this theory as phenomenological LRT. It has been shown $[14,15]$ that the choice of Eq. (16), called dynamic LRT, corresponds to the response being produced by the external perturbation affecting the event occurrence time according to the state of the system $S$.

The dynamic LRT yields [30]

$$
\Lambda^{-}(t)=\frac{\epsilon}{\Gamma(\mu-1)} \frac{\cos \left(\frac{\pi \mu}{2}+\omega t\right)}{(\omega t)^{2-\mu}} .
$$

This result corresponds to the survival probability of Eq. (12) and to the strict assumption that $\xi_{S}(t)$ is dichoto- 


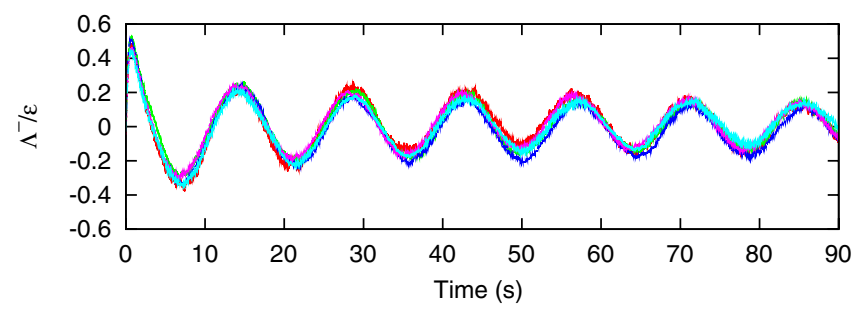

FIG. 3 (color online). $\quad \Lambda^{-}(t) / \epsilon$ with $\omega / 2 \pi=0.07 \mathrm{~Hz}$ and $\epsilon=$ $0.025,0.031,0.037,0.044,0.050$.

mous. We have established that survival probabilities departing from Eq. (12) in the short-time limit and a nonstrictly dichotomous $\xi_{S}(t)$ affect the values of $\phi$ and $C$. Thus, to keep the focus of this Letter on the universal structure of Eq. (4), we consider both $\phi$ and $C$ as fitting parameters.

We notice that the phenomenological theory would generate a correction to Eq. (2) under the form of a slow decay process proportional to $1 / t^{\mu-1}$. This correction depends on the first instants of the perturbation, and it would be positive for $\left\langle\xi_{S}(t)\right\rangle_{+}$and negative for $\left\langle\xi_{S}(t)\right\rangle_{-}$[34]. Figure 2, where $\Lambda^{-}$oscillates around zero, does not reveal any sign of this effect, thereby proving that the dynamic LRT is in action in this case.

To complete the experimental proof of the nonstationary LRT, we generate several perturbations with different intensity, rescaling the results by perturbation amplitude. The result is illustrated by Fig. 3: Curves are superimposed one to the others, thereby proving that for small perturbations $(\epsilon \lesssim 0.05)$, the response of the system is linear with respect to the perturbation intensity.

We notice that the empirical prescription of Eq. (4) is compatible with both Eq. (8) and, for $\mu>1.5$, with Eq. (7) as well. The main conclusion is that the LRT is not dead, and that rather it is a universal property of nature, valid in both the case of Hamiltonian and stationary statistical physics and in the challenging case of event-dominated dynamics. The response of a complex system to a simple perturbation fades away as a consequence of the fact that a simple stimulus does not match the system's complexity. According to the Complexity Matching Principle [21], the event-dominated LRT is expected to be compatible with a steady response to a proper complex stimulus. The experimental result of this Letter is therefore an encouraging indication that the event-dominated LRT may have application of surprising generality and of great interest, such as the explanation of why the brain, as a complex system, is sensitive to art [35] as a source of intellectual stimuli.

P.G. and M.B. thank ARO and Welch for financial support of this work through Grants No. W911NF-0810177 and No. B-1577, respectively.
[1] R. Kubo, Can. J. Phys. 34, 1274 (1956); R. Kubo, M. Toda, and N. Hashitsume, Statistical Physics II (Springer, Berlin, 1985).

[2] N. G. van Kampen, Phys. Norv. 5, 279 (1971).

[3] M. H. Lee, Phys. Rev. Lett. 49, 1072 (1982).

[4] M. H. Lee, Phys. Rev. Lett. 98, 190601 (2007).

[5] U. Balucani, M. H. Lee, and V. Tognetti, Phys. Rep. 373, 409 (2003).

[6] F. Corberi, E. Lipiello, and M. Zannetti, J. Stat. Mech. (2007) P07002.

[7] P. Hänggi and H. Thomas, Phys. Rep. 88, 207 (1982).

[8] X. Brokmann et al., Phys. Rev. Lett. 90, 120601 (2003).

[9] G. Margolin and E. Barkai, J. Stat. Phys. 122, 137 (2006); Phys. Rev. Lett. 94, 080601 (2005).

[10] A. Rebenshtok and E. Barkai, J. Stat. Phys. 133, 565 (2008).

[11] H.-P. Breuer and B. Vacchini, Phys. Rev. E 79, 041147 (2009).

[12] P. Gong, A. R. Nikolaev, and C. van Leeuwen, Phys. Rev. E 76, 011904 (2007).

[13] M. Bologna and P. Grigolini, J. Stat. Mech. (2009) P03005.

[14] P. Allegrini et al., Phys. Rev. Lett. 99, 010603 (2007).

[15] G. Aquino, P. Grigolini, and B. J. West, Europhys. Lett. 80, 10002 (2007).

[16] I. M. Sokolov and J. Klafter, Chaos Solitons Fractals 34, 81 (2007).

[17] I. M. Sokolov and J. Klafter, Phys. Rev. Lett. 97, 140602 (2006).

[18] E. Heinsalu et al., Phys. Rev. Lett. 99, 120602 (2007).

[19] M. Magdziarz, A. Weron, and K. Weron, Phys. Rev. E 75, 016708 (2007).

[20] M. Magdziarz, A. Weron, and J. Klafter, Phys. Rev. Lett. 101, 210601 (2008).

[21] B. J. West, E. Geneston, and P. Grigolini, Phys. Rep. 468, 1 (2008).

[22] W. Feller, An Introduction to Probability Theory and Its Applications (John Wiley \& Sons, New York, 1971), Vol. II.

[23] M. Luković et al., Phys. Lett. A 372, 2608 (2008).

[24] J. T. Gleeson, Phys. Rev. E 63, 026306 (2001); V. Carbone et al., Phys. Rev. E 54, 6948 (1996); T. Tóth-Katona and J. T. Gleeson, Phys. Rev. Lett. 91, 264501 (2003).

[25] P.G. de Gennes and J. Prost, The Physics of Liquid Crystals (Clarendon Press, Oxford, 1993).

[26] R. Williams, J. Chem. Phys. 39, 384 (1963).

[27] Example movies of the defects dynamics available at http://mail.df.unipi.it/ allegrip/liquid_crystal.html.

[28] T. Tóth-Katona and J. T. Gleeson, Phys. Rev. E 69, 016302 (2004).

[29] L. Silvestri et al., Phys. Rev. Lett. 102, 014502 (2009).

[30] F. Barbi, M. Bologna, and P. Grigolini, Phys. Rev. Lett. 95, 220601 (2005).

[31] D. R. Cox, Renewal Theory (Methuen, London, 1962).

[32] P. Allegrini et al., Phys. Rev. E 71, 066109 (2005).

[33] I. M. Sokolov, Phys. Rev. E 73, 067102 (2006).

[34] P. Allegrini et al., arXiv:cond-mat/0602281.

[35] I. Yevin, Complexus 3, 74 (2006). 